\title{
Secoisolariciresinol Diglucoside Regulates Adipose Tissue Metabolic Disorder in Obese Mice Induced by a Western Diet
}

\author{
Shan Dong $\left(\mathbb{D},{ }^{1}\right.$ Wenliang Bai, ${ }^{1}$ Jiaping Chen, ${ }^{1}$ Li Zhang, ${ }^{2}$ Wanli Sheng, ${ }^{3}$ and Ronghu Feng ${ }^{1}$ \\ ${ }^{1}$ Shenzhen Academy of Metrology and Quality Inspection, National Nutrition Food Testing Center, Shenzhen 518055, China \\ ${ }^{2}$ College of Food Science and Engineering, Northwest A\&F University, Yangling 712100, China \\ ${ }^{3}$ Technical Center of Hohhot Customs District, Huhehaote 010000, China
}

Correspondence should be addressed to Shan Dong; 2810558181@qq.com

Received 5 February 2021; Revised 17 March 2021; Accepted 24 March 2021; Published 2 April 2021

Academic Editor: quancai sun

Copyright ( $\odot 2021$ Shan Dong et al. This is an open access article distributed under the Creative Commons Attribution License, which permits unrestricted use, distribution, and reproduction in any medium, provided the original work is properly cited.

Secoisolariciresinol diglucoside (SDG) is the main component of flax lignans. Current studies have reported a positive effect of SDG on obesity and metabolic diseases. SDG has strong blood fat- and blood sugar-lowering, anti-inflammatory, and antioxidant effects and prevents heart disease and other chronic diseases. In this study, we explored the effects of SDG on Western dietinduced obesity and lipid metabolic disorder. Supplementing Western diet-induced obese mice with $40 \mathrm{mg} \mathrm{kg}^{1} \mathrm{~d}^{1}, \mathrm{SDG}_{\text {for }} 12$ weeks significantly reduced body and tissue weights. Increased adiponectin levels and decreased serum leptin and resistin levels were observed in obese mice orally administered SDG. Proliferation of adipose tissue was observed by hematoxylin and eosin staining, and cell size was quantitatively analyzed. As a result, SDG inhibited the proliferation of adipose tissue. In addition, SDG suppressed the mRNA expression of lipid synthetic genes and upregulated the mRNA expression of lipolytic genes. Overall, these results indicate that SDG inhibits obesity induced by a Western diet and regulates adipose tissue metabolic disorder. These results provide a theoretical basis for further study on the regulation of obesity and lipid metabolic disorder caused by SDG.

\section{Introduction}

Obesity is a chronic state of excessive accumulation of body fat. A positive correlation has been reported between obesity and metabolic diseases, including diabetes, hypertension, and hyperlipidemia [1]. An imbalance in the amount of energy consumed and expended is the main cause of obesity [2]. When caloric intake exceeds the amount expended, adipocyte hypertrophy and mature hyperplastic adipocytes are produced, resulting in increased fat mass [3]. The diet has gradually shifted to a Western diet high in sugar and fat, which has led to a rapid rise in the incidence of obesity [4]. Therefore, obesity has become a global health problem that cannot be ignored [5].

Obesity is characterized by excessive adipose tissue accumulation, triglyceride accumulation, and prominent adipocyte hypertrophy, resulting in disrupted adipose tissue homeostasis and adipocyte cell death, which are important in lipid metabolic disorder [6]. Lipid metabolic disorder refers to the imbalance between anabolism and catabolism of lipids, which causes the accumulation of lipids in various tissues, thus affecting metabolic balance [7]. Adipose tissue increases in mass and quantity in obesity with a significant variation in its metabolic and immunological profile. This variation leads to lytic death of adipocytes and triggers the release of free fatty acids and several metabolites, thus transforming the immune repertoire into a proinflammatory state. This results in abnormal cytokine secretion, which promotes heterotopic fat accumulation and low-grade inflammation, ultimately exacerbating liver steatosis and insulin resistance [8-10]. In addition, upregulation of several inflammatory genes in immune cells drives the inflammatory response in adipose tissue, leading to insulin resistance and glucose intolerance [11]. Therefore, variations in metabolism and inflammation in adipose tissue destroy physiological homeostasis in tissues and systems by directly impacting the development of obesity and the initiation and 
progression of cancer and metabolic syndrome and creating a positive feedback loop between them [12].

Adipokines are biologically active cytokines secreted by adipocytes that play important regulatory roles in insulin resistance, inflammatory response, and glucose and lipid metabolism [13]. Adiponectin is an important adipokine that increases insulin sensitivity, promotes healthy expansion of adipose tissue, and improves ectopic fat deposition [14]. Studies have shown that human and rodent serum levels of adiponectin decrease in insulin resistance and type 2 diabetes or obesity, and that increased levels of adiponectin inhibit the accumulation of hepatic lipids in obese animals induced by a high-fat diet [15]. Leptin, or antiobesity factor, is an appetite-related adipose factor that affects lipid metabolism by regulating food intake through the hypothalamus $[16,17]$. Leptin receptors are widely expressed in many tissues and the level of circulation is proportional to the amount of fat. It has been reported that leptin reduces fat production and increases triglyceride hydrolysis and fatty acid oxidation [18].

tIn recent years, the antiobesity function of natural products has been gradually explored and studied. Among them, researchers pay more attention on polyphenols, which are derived from natural plants such as fruits and vegetables $[19,20]$. Studies showed that polyphenols such as quercetin, curcumin, and resveratrol exerted beneficial effects on lipid and energy metabolism and potential body weight change [21-23]. Quercetin is the most abundant of flavonoids which is found naturally in vegetables, fruits, and tea [24]. Animal studies showed that quercetin can protect mice or rats from high-fat diet-induced body weight gain and adipose tissue accumulation $[25,26]$. In another study, quercetin $(10 \mathrm{mg} / \mathrm{kg}$ of body weight) improved the inflammatory status of visceral adipose tissue by suppressing the expression of TNF- $\alpha$ and enhancing the levels of adiponectin, which indicates the recovery of the functions of the adipose tissue, in obese Zucker rats, a genetically obese rat model [27]. Curcumin is derived from the spice turmeric which is the most bioactive polyphenol. Curcumin exerts several biological functions including antioxidation, anti-inflammation, and antiangiogenesis in different organs including adipose tissue [28]. Curcumin showed beneficial effects on energy metabolism and body weight reduction. Two weeks of high dietary curcumin supplementation feeding in rats reduced epididymal adipose tissue and increased fatty acid $\beta$-oxidation, indicating the increase of energy expenditure after curcumin treatment [29]. Resveratrol is a polyphenolic compound, which showed beneficial effects in preventing the development of many diseases including obesity and diabetes [30-32]. Dietary treatment of rodents with resveratrol protected mice against HFD-induced body weight gain and obesity by increasing energy expenditure which was partly mediated by stimulating intracellular mitochondrial functions in adipose tissue and by the suppression of fatty acid synthesis [33-35].

Secoisolariciresinol diglucoside (SDG) is the main form of flax lignan in flaxseed, which has beneficial effects in diseases, such as diabetes, hypertension, cardiovascular disease, and obesity [36]. The structure of SDG is shown in Figure 1. Studies have shown that SDG reduces liver and serum lipid levels, inhibits excess accumulation of visceral lipids, and improves glucose tolerance, insulin resistance, and liver steatosis in obese mice [37-40]. These reports have demonstrated that SDG effectively improves obesity. However, few reports are available on the SDG mechanism of regulating lipid metabolic disorder. Therefore, in this study, adipose tissue was targeted to explore the regulatory effects of SDG on lipid metabolic disorder in Western dietinduced obese mice, and the related mechanisms were explored.

\section{Materials and Methods}

2.1. Animal Experiments. Male C57BL/6J mice (age 8 weeks) were purchased from Xian Jiaotong University (Xi 'an, Shaanxi, China) and raised in a standard environment $(12 \mathrm{~h} /$ $12 \mathrm{~h} \mathrm{light/dark}$ cycle, temperature $25 \pm 2^{\circ} \mathrm{C}$, humidity $50 \pm 5 \%$ ), and were given free access to feed and drinking water. All animal experiments followed the Guidelines for the Protection and Use of Experimental Animals (8th Edition) and the Laboratory Animal Management Measures of Northwest A\&F University. This animal protocol was approved by the Animal Ethics Committee of Xian Jiaotong University. AIN-93M standard feed used in the experiment (LAD3001M, calorie value $3.6 \mathrm{kcal} / \mathrm{g}$ ) and the $45 \%$ high-fat feed (TP230100, calorie value $4.5 \mathrm{kcal} / \mathrm{g}$ ) were purchased from Nantong Troffer Feed Technology Co., Ltd. (Nantong, Jiangsu, China). SDG (>95\%) was purchased from Chengdu Biopurify Phytochemicals Ltd. (Chengdu, China). Fructose (99\%) was purchased from Sigma-Aldrich (St. Louis, MO, USA). The mice were randomly divided into four groups (10/ group) and fed a different diet for 12 weeks: control (standard diet, AIN-93M, containing $3.6 \mathrm{kcal} / \mathrm{g}$; Trophic Animal Feed High-Tech Co., Ltd., Nantong, China; drinking purified water), control + SDG (40 $\mathrm{mg} \mathrm{kg}^{-1} \mathrm{~d}^{-1} \mathrm{SDG}$, mixed with the AIN-93M diet; drinking purified water), HFFD (45\% kcal from fat, TP230100, containing $4.5 \mathrm{kcal} / \mathrm{g}$ and $10 \%$ fructose in drinking water), and the HFFD + SDG groups (40 $\mathrm{mg} \mathrm{kg}^{-1} \mathrm{~d}^{-1}$ SDG, mixed with the TP230100 diet and 10\% fructose in drinking water). The feed composition is shown in Tables 1 and 2, respectively.

2.2. Hematoxylin-Eosin Staining. The white adipose tissue was fixed in $4 \%$ formalin, paraffin-embedded, and cut into 5$\mu \mathrm{m}$ thick slices, respectively, in xylene I and II, dewaxed for $10 \mathrm{~min}$, and then run through in gradient ethanol series (100\% I and II, 90\% I and II, 80\%, 70\%) and rinsed in double distilled water for $5 \mathrm{~min}$. After rinsing with PBS (3 times, $5 \mathrm{~min}$ each), hematoxylin stain was used for $3 \mathrm{~min}$, and $1 \%$ acetic acid differentiation solution was used to separate the color for $15 \mathrm{~s}$. After rinsing with running water, the sections were placed in $1 \%$ dilute ammonia for $5-10 \mathrm{~s}$ and then dyed with eosin solution for $3 \mathrm{~min}$. They were dehydrated through a gradient ethanol series $(70 \%, 80 \%, 90 \%$, and $100 \%)$ for $1 \mathrm{~min}$ each treated with xylene and observed under an optical microscope. 


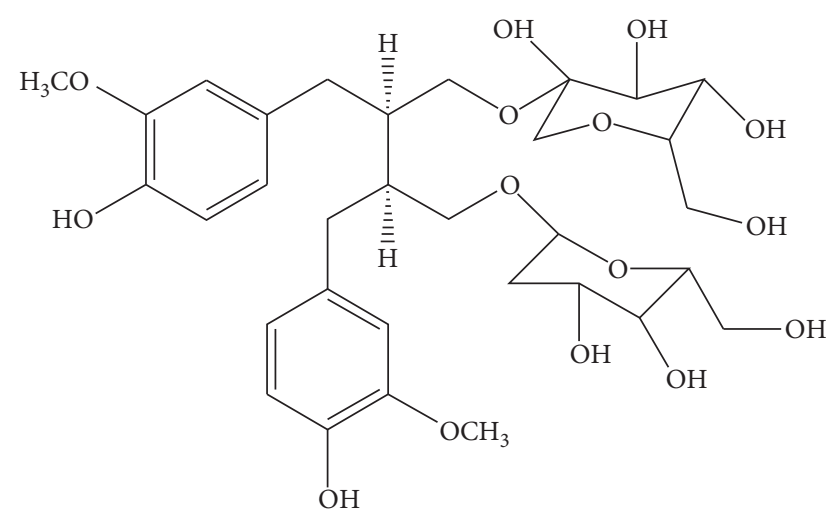

Figure 1: Structure of SDG.

TABle 1: Composition of nutrients of standard diet.

\begin{tabular}{lc}
\hline Nutrients & Content \\
\hline Corn starch & 465.7 \\
Maltodextrin & 155 \\
Sucrose & 100 \\
Soybean oil & 40 \\
Mineral mix, M1021 & 35 \\
Casein & 140 \\
TBHQ & 0.036 \\
Choline chloride & 2.5 \\
Cellulose & 50 \\
Vitamin mix, V1010 & 10 \\
L-Cystine & 1.8 \\
Total & 1000 \\
\hline
\end{tabular}

TABLE 2: Composition of nutrients of high-fat diet.

\begin{tabular}{lc}
\hline Nutrients & Content \\
\hline Corn starch & 132 \\
Maltodextrin & 125 \\
Choline bitartrate & 3 \\
Sucrose & 202 \\
Soybean oil & 30 \\
Lard & 196 \\
Mineral mix, M1021 & 61 \\
TBHQ & 0.045 \\
Casein & 175 \\
Vitamin mix, V1010 & 12 \\
Cellulose & 62 \\
L-Cystine & 2 \\
Total & 1000 \\
\hline
\end{tabular}

2.3. Serum Adipokines. After the mice were sacrificed, eyeball blood was extracted, plasma was separated, and the plasma levels of adiponectin, leptin, and resistin were detected according to the manufacturer's instructions for the enzyme-linked immunosorbent assay kit.

2.4. Quantitative Real-Time PCR Analysis. Total RNA was extracted from mouse adipose tissue with TRIZOL according to the manufacturer's instructions (TransGen
Biotech, Beijing, China) quantified with a nucleic acid quantifier (OD260/280) and diluted to a uniform concentration. The samples were reverse-transcribed to cDNA according to the HiFiScript gDNA Removal RT MasterMix instructions. Reverse transcriptional products were treated according to the manufacturer's method described in the SYBR kit. mRNA expression of the treated samples was detected. The relative expression of genes was calculated with the $2^{-\triangle \triangle \mathrm{Ct}}$ method using GAPDH as the internal reference. The primer sequences are shown in Table 3.

2.5. Statistical Analysis. Data are expressed as mean\pm standard error. Significant differences between the groups were determined by two-way analysis of variance followed by Tukey's test. The statistical analysis was performed with GraphPad Prism 6 software (GraphPad Software Inc., La Jolla, CA, USA). A $p$ value $<0.05$ was considered significant.

\section{Results and Discussion}

3.1. SDG Inhibits Weight Gain in HFFD-Fed Mice. The mice were divided into four groups during the 12-week diet intervention: control (normal diet), HFFD (high-fat and fructose diet), SDG (SDG mixed with the normal diet), and HFFD + SDG (SDG mixed with high-fat and fructose diet) groups. As shown in Figure 2(b), mice in the HFFD group increased their body weight by $9.7 \%$ compared to that in the control group $(p<0.05)$, which lasted until week $12(21.9 \%$, $p<0.01)$. The SDG treatment significantly suppressed weight gain in the obese mice from week $7(9.3 \%, p<0.05)$ to week $12(13.3 \%, p<0.01$; Figure $2(\mathrm{~b}))$. The progression of obesity is related to the ratio of tissue weight to body weight. The percentage of tissue weight in the mice was analyzed after sacrifice, and the results showed that the liver and adipose tissue ratio increased in the HFFD group compared with the control group $(p<0.05)$, while the percentage decreased significantly in response to the SDG supplement $(p<0.05$; Figure 2(c)).

\subsection{SDG Regulates Serum Adipokines in Obese Mice Induced} by a Western Diet. Adipokines are closely related to the pathogenesis of obesity. Leptin promotes food intake, thereby regulating the energy and weight balance of the body. The serum leptin content in the HFFD group increased significantly $(p<0.01)$ compared to that in the control group, and SDG notably reduced this trend $(p<0.01$; Figure 3(a)). High resistin levels reduce insulin sensitivity. Our results show that the HFFD group presented a significantly higher serum resistin level compared with that in the control group. However, the SDG treatment reversed this effect $(p<0.01$; Figure 3(c)). Similarly, adiponectin levels also reflect the state of insulin resistance in the body. Figure 3(b) shows that the adiponectin level in the HFFD group decreased significantly compared with that in the control group $(p<0.01)$, and administration of SDG reversed this trend $(p<0.01)$. 
TABle 3: Primer sequences for RT-PCR (all primers are mouse primers).

\begin{tabular}{lcc}
\hline & Forward primers & Reverse primers \\
\hline Srebplc & AAGCAAATCACTGAAGGACCTGG & AAAGACAAGGGGCTACTCTGGGAG \\
Fasn & AGGTGGTGATAGCCGGTATGT & TGGGTAATCCATAGAGCCCAG \\
PpAR $\gamma$ & TGCTGTTATGGGTGAAACTCTG & CTGTGTCAACCATGGTAATTTCTT \\
Acaca & CCGATTCATAATTGGGTCTGTGT & CCATCCTGTAAGCCAGAGATCC \\
PpAR $\alpha$ & ACAGGAGAGCAGGGATTTGC & TACCTACGCTCAGCCCTCTT \\
Pgcl $\alpha$ & GAAAGGGCCAAACAGAGAGA & GTAAATCACACGGCGCTCTT \\
GAPDH & TGGAGAAACCTGCCAAGTATGA & TGGAAGAATGGGAGTTGCTGT \\
\hline
\end{tabular}

3.3. SDG Alleviates Adipose Tissue Hyperplasia Induced by a Western Diet in Obese Mice. Obesity is associated with excessive accumulation of lipids in tissues, which leads to increased adipose tissue, an increase in the number of adipose cells, and expansion in particle size and area. To investigate the effect of SDG on the proliferation of adipose tissue in HFFD-induced obese mice, the morphology of epididymal white adipose tissue (eWAT), subcutaneous white adipose tissue (iWAT), and brown adipose tissue (BAT) was observed by hematoxylin and eosin (H\&E) staining, and the area of adipose tissue cells was quantitatively analyzed (Figure 4). The adipose tissue cells in the HFFD group were dilatated, and the dilatation was significantly inhibited by SDG (Figure 4(a)). The analysis of adipocyte area further confirmed this effect. The mean adipocyte area of eWAT, iWAT, and BAT cells in the HFFD group increased compared to that in the control group $(p<0.01)$, but this increase was suppressed after SDG was administered $(p<0.01$; Figure 4$)$. In addition, supplementing with SDG had no effect on morphology or cell area of adipose tissue in normal mice. These results indicate that oral administration of SDG inhibits the accumulation of lipids in adipose tissue of HFFD-induced obese mice.

3.4. SDG Regulates Lipid Metabolic Genes in White Adipose Tissue of Obese Mice Induced by a Western Diet. To investigate the mechanism of SDG regulating lipid metabolic homeostasis in adipose tissue, the mRNA expression of genes involved in lipogenesis and lipolysis in eWAT was evaluated by real-time polymerase chain reaction analysis. As shown in Figure 5, the expression of genes associated with lipogenesis, such as Srebplc, Fasn, Ppar, and Acaca, in mice of the HFFD group were significantly upregulated $(p<0.05$; Figures 5(a)-5(d)), and the expression levels of Ppar and Pgc1 were downregulated ( $p<0.05$; Figures 5(e) and 5(f)). SDG reversed this tendency. These results suggest that SDG improves lipid metabolic disorder in HFFD-induced obese mice by regulating the mRNA expression levels of lipidrelated genes.

3.5. Discussion. The results of this study show that SDG significantly improved obesity and regulated the lipid metabolic disorder induced by a Western diet: SDG suppressed body weight gain and tissue weight in obese mice; SDG regulated the energy and body weight balance by regulating serum adipokines; SDG reduced the proliferation of adipose tissue cells in mice and inhibited excessive accumulation of lipids; SDG improved the lipid metabolic disorder caused by obesity by promoting the expression of lipolytic genes and inhibiting the expression of lipogenic genes.

Flaxseed is the mature seed of flax, which is rich in fat, protein, and dietary fiber, as well as other bioactive components, such as alpha-linolenic acid, lignans, and vitamins. Flaxseed is a dietary source containing high-value functional components, mainly used to produce and process edible vegetable oil seeds $[41,42]$. A number of studies have shown that flaxseed has strong blood fat- and blood sugar-lowering, anti-inflammatory, and antioxidant effects and prevents heart disease and other chronic diseases [43, 44]. Flaxseed lignan is an important bioactive ingredient of flaxseed. Its main form is SDG. Dietary intake of SDG has positive effects on a variety of diseases, including cancers, cardiovascular diseases, diabetes, obesity, and metabolic syndrome [36]. Fukumitsu et al. reported that adding $0.1 \%$ or $0.5 \%$ SDG to a high-fat diet significantly reduces liver triglycerides, total cholesterol levels, and serum insulin and leptin levels in C57BL/6 mice, indicating that SDG improves diet-induced obesity and insulin resistance [45]. In a clinical study, Zhang et al. reported that a daily supplement of $600 \mathrm{mg}$ SDG for 8 weeks in patients with hypercholesterolemia reduces plasma total cholesterol, low-density lipoprotein cholesterol, and fasting blood glucose levels [46]. Kang et al. reported that supplementation with SDG $(50 \mathrm{mg} / \mathrm{kg} / \mathrm{d})$ inhibits fat formation in obese mice induced by a high-fat diet by reducing Ppar protein expression [47]. Pan et al. further demonstrated the beneficial effects of SDG in a randomized, double-blind trial of 68 patients with type 2 diabetes who were given $360 \mathrm{mg}$ of SDG daily for 12 weeks. The results showed that indicators related to insulin resistance, type 2 diabetes, and inflammation, such as C-reactive protein, improved [48]. Based on these results, the dosage of SDG ( $40 \mathrm{mg} / \mathrm{kg} / \mathrm{d})$ used in this study was safe and effective. The body and tissue weights of obese mice induced by a Western diet were significantly inhibited in response to this SDG dose for 12 weeks, which improved obesity induced by the Western diet (Figures 2(b) and 2(c)).

As a chronic metabolic disease, obesity causes hypertension, diabetes, hyperlipidemia, coronary heart disease, and other diseases, which seriously threaten human health. According to the World Health Organization, the number of obese people worldwide increased nearly threefold between 1975 and 2016. By 2018, the number of obese people worldwide was still on the rise, reaching 672 million $[4,5]$. At present, the main cause of obesity is the change in the 

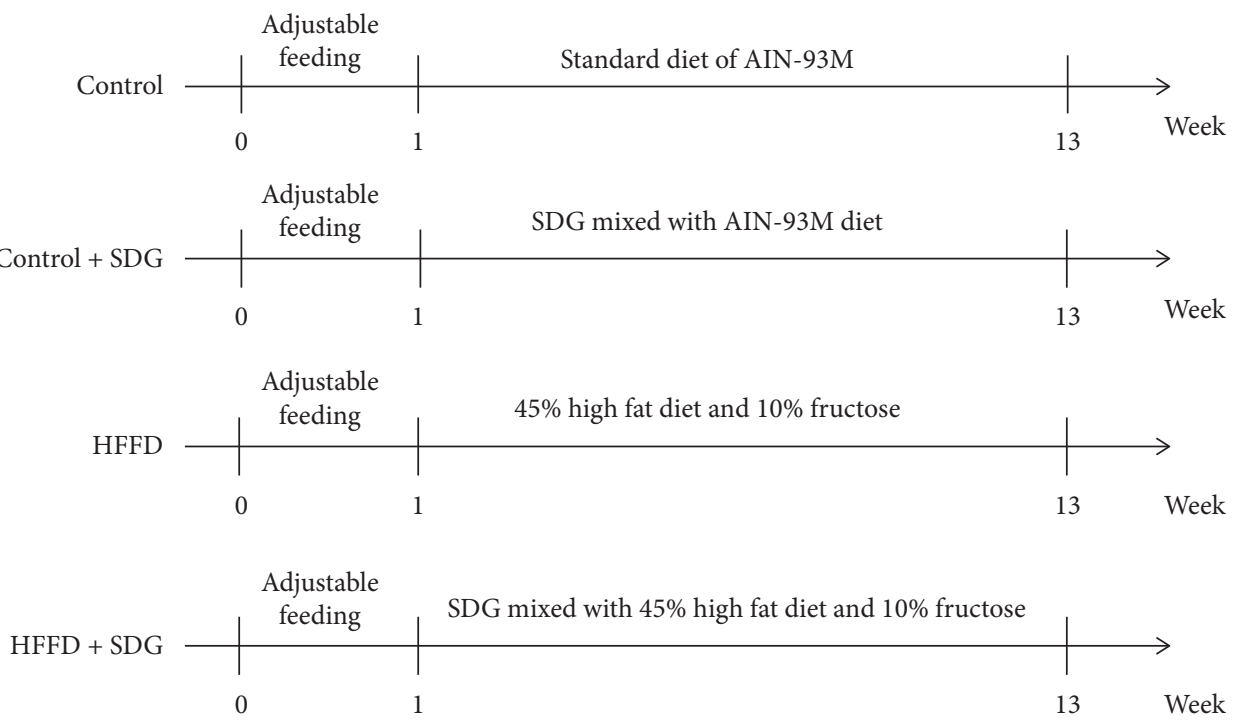

(a)

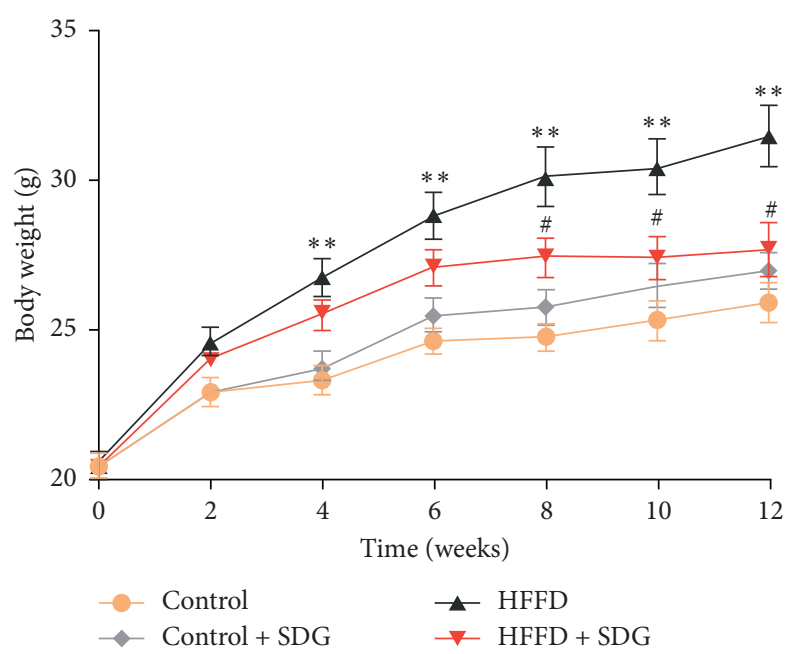

(b)

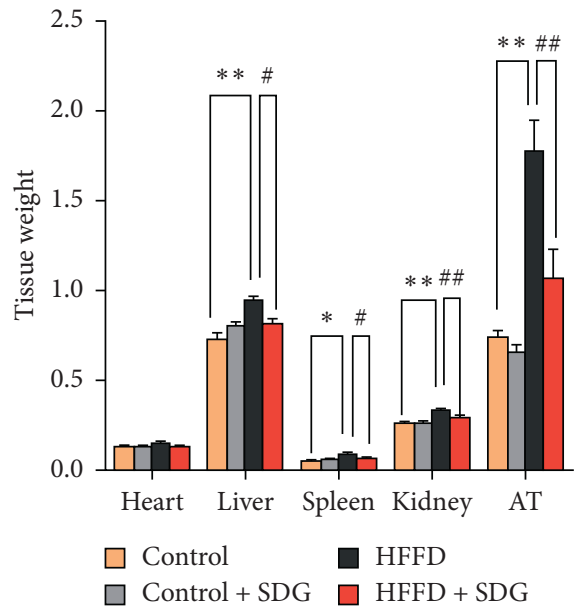

(c)

FIGURE 2: SDG inhibits weight gain in HFFD-fed mice. (a) Test design and groups; (b) trends in body weight during feeding and (c) tissue weight to body weight ratio (heart, liver, spleen, kidney, adipose tissue (AT)). Data are represented as mean \pm SEM, $n=10$ biologically independent animals. ${ }^{*} p<0.05,{ }^{* *} p<0.01$ versus the control group; ${ }^{\#} p<0.05,{ }^{\#} p<0.01$ versus the HFFD group. 


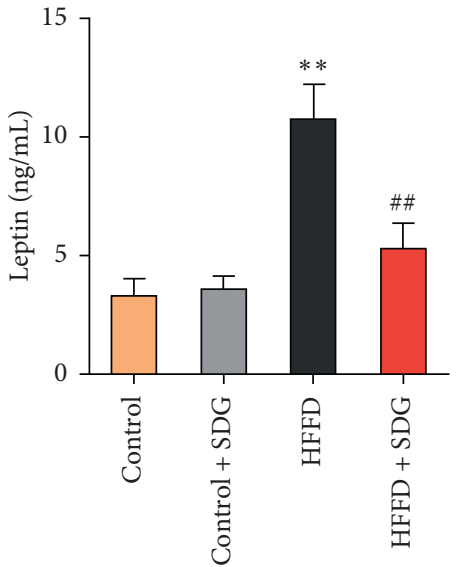

(a)

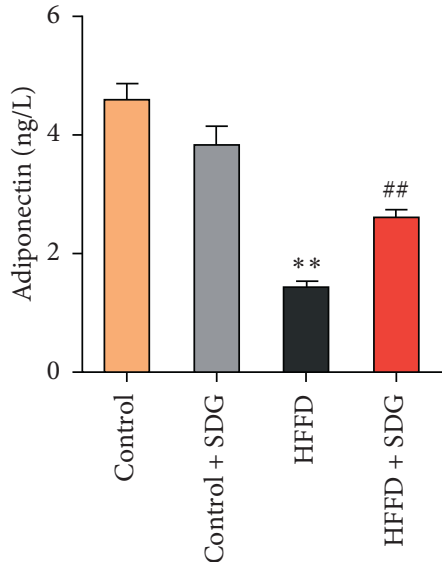

(b)

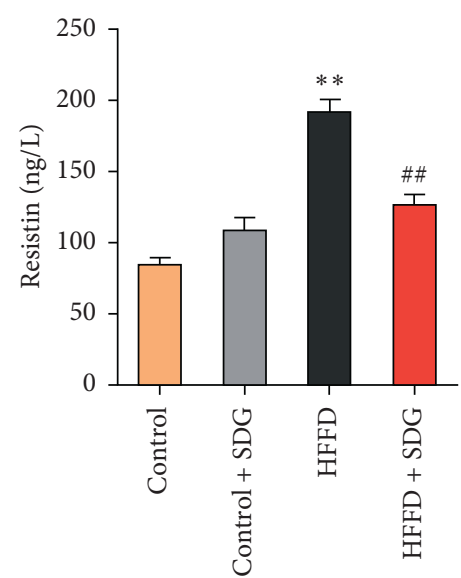

(c)

FIGURE 3: SDG regulates serum adipokines in obese mice induced by a Western diet. (a) Leptin; (b) adiponectin, and (c) resistin. Data are represented as mean \pm SEM, $n=10$ biologically independent animals. ${ }^{*} p<0.05,{ }^{* *} p<0.01$ versus the control group; ${ }^{\#} p<0.05,{ }^{\# \#} p<0.01$ versus the HFFD group.

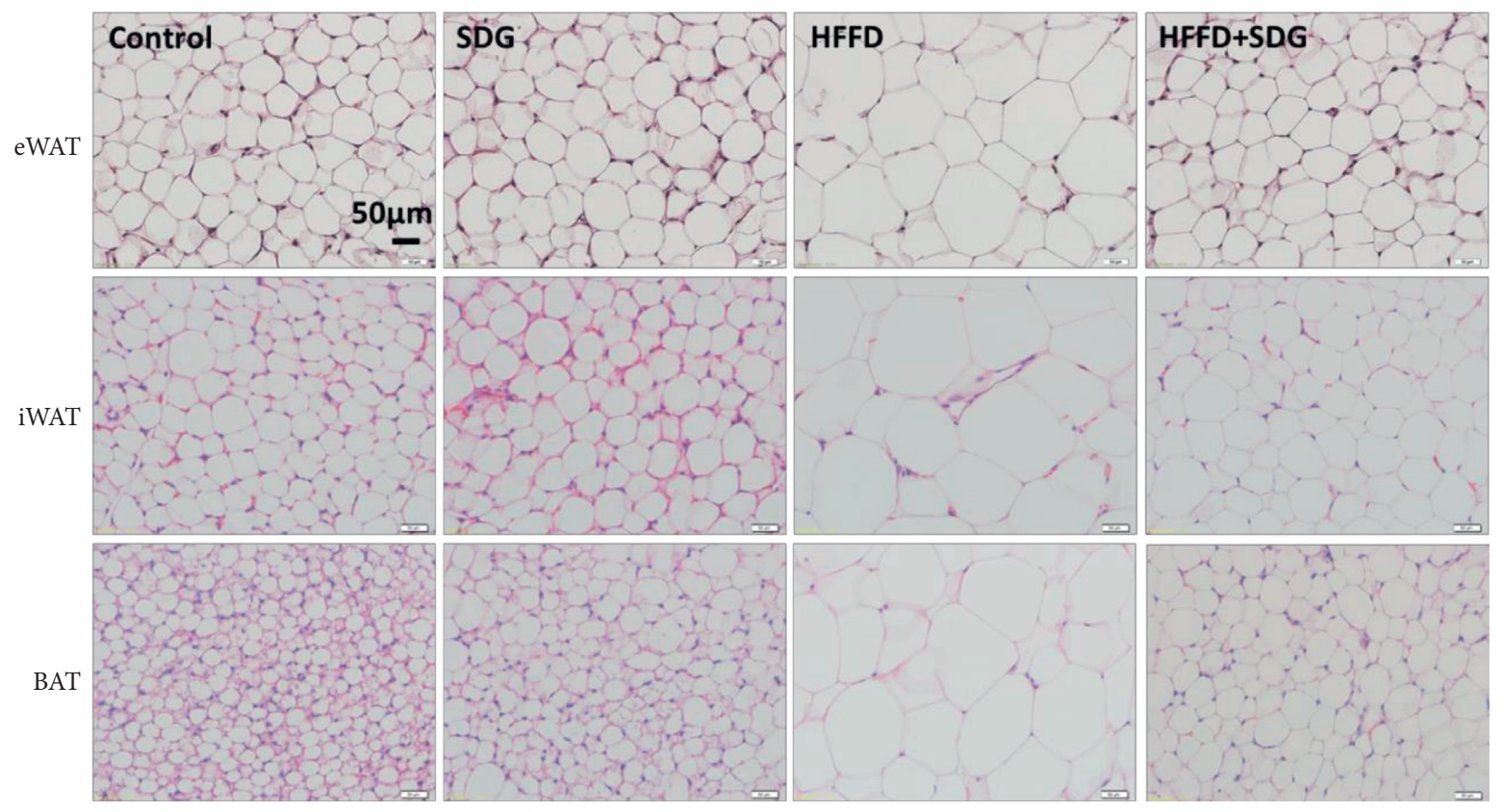

(a)

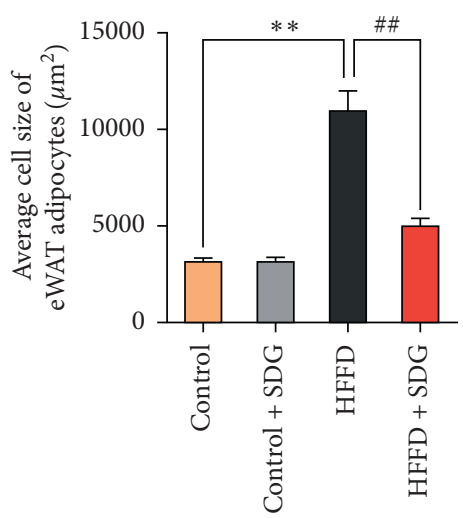

(b)

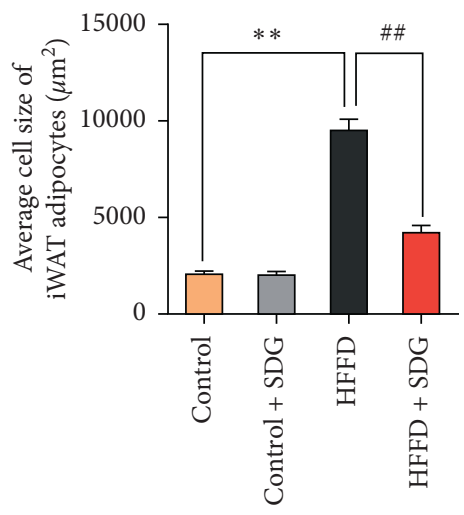

(c)

Figure 4: Continued. 


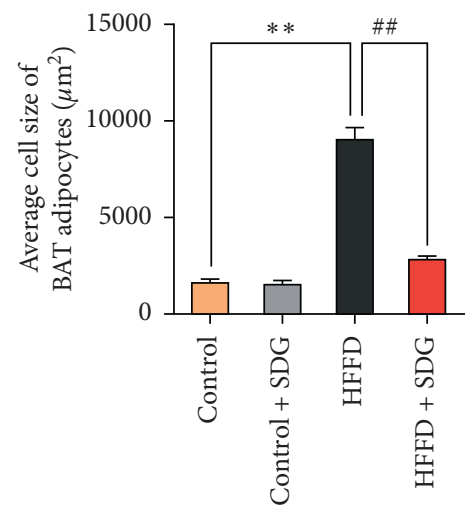

(d)

FigURE 4: SDG alleviates adipose tissue hyperplasia induced by a Western diet in obese mice. (a) H\&E staining ( $\times 200)$; (b) average area of white adipose tissue cells in the epididymis; (c) average area of subcutaneous white adipose tissue cells; and (d) average area of brown adipose tissue cells. Data are represented as mean \pm SEM, $n=10$ biologically independent animals. ${ }^{*} p<0.05,{ }^{* *} p<0.01$ versus the control group; ${ }^{\#} p<0.05,{ }^{\# \#} p<0.01$ versus the HFFD group.

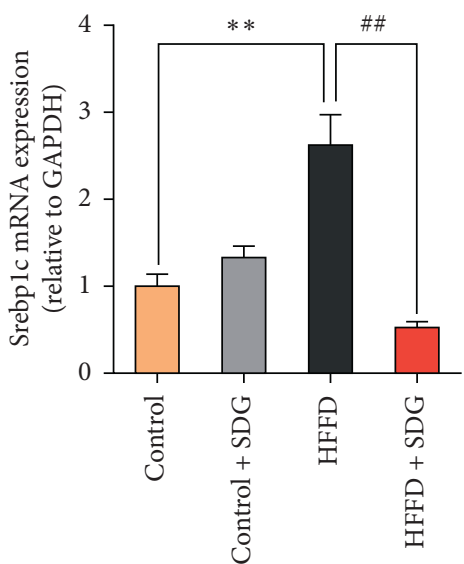

(a)

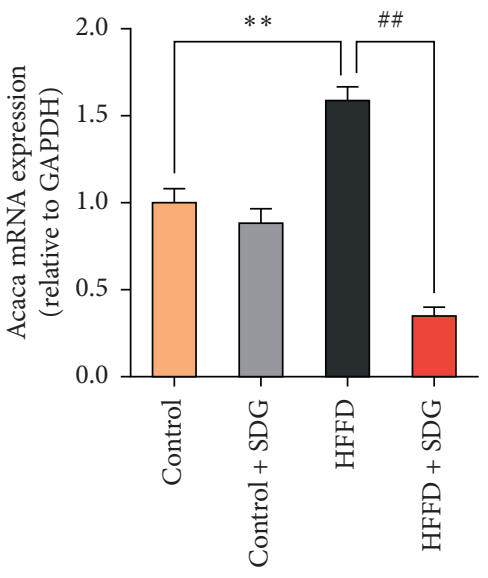

(d)

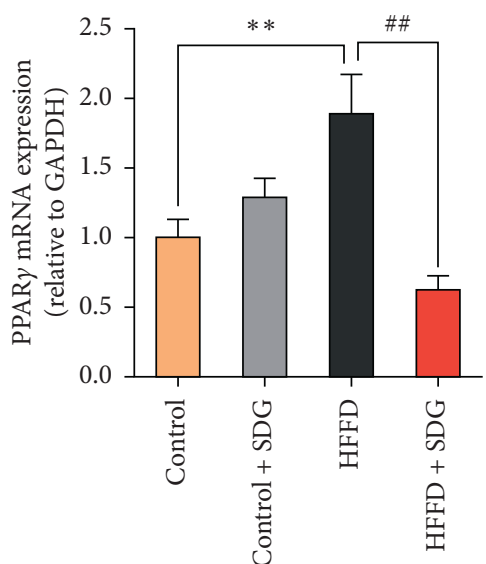

(b)

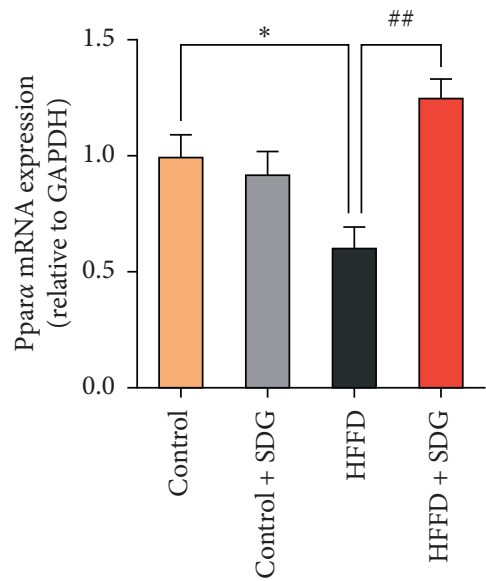

(e)

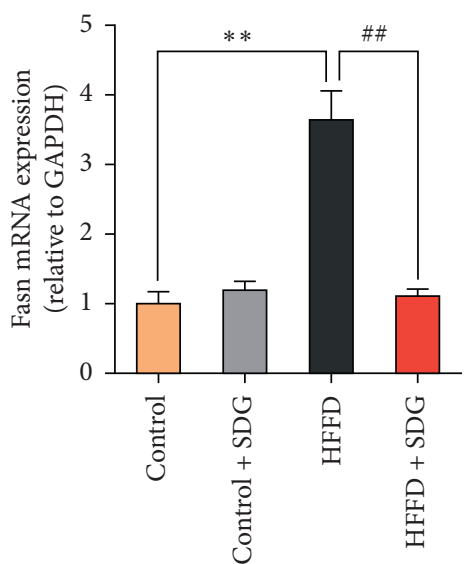

(c)

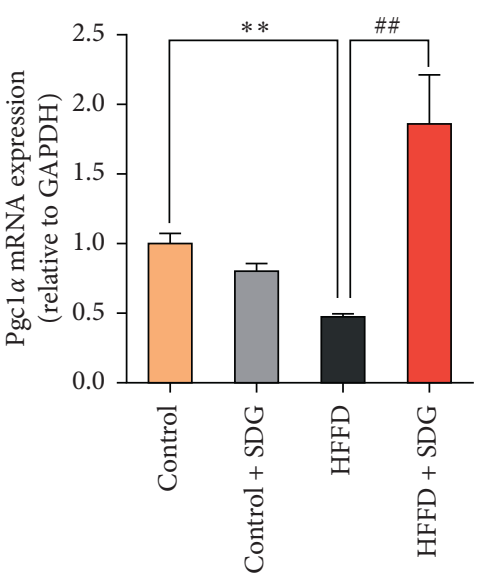

(f)

FIGURE 5: SDG regulates lipid metabolic genes in white adipose tissue of obese mice induced by a Western diet. (a) Srebplc mRNA level; (b) PPAR $\gamma$ mRNA level; (c) Fasn mRNA level; (d) Acaca mRNA level; (e) Ppar $\alpha$ mRNA level; and (f) Pgcl mRNA level. Data are represented as mean \pm SEM, $n=10$ biologically independent animals. ${ }^{*} p<0.05,{ }^{* *} p<0.01$ versus the control group; ${ }^{\#} p<0.05$, ${ }^{\# \#} p<0.01$ versus the HFFD group. 
dietary pattern. A Western diet with high sugar and fat has gradually become popular. People take in more energy than the body needs, which leads to obesity. One of the metabolic diseases closely associated with obesity is diabetes. Obese adults increased their risk of developing diabetes by about one-tenth between 1960 and 2000 [49]. The most common form of diabetes is type 2 diabetes (T2DM). T2DM is often accompanied by insufficient insulin secretion or insulin resistance, while obesity leads to the deposition of ectopic fat and the proliferation of adipose tissue cells, which causes insulin resistance. Adipose tissue participates in the circulation of fatty acids by regulating lipid metabolism. Fatty acids are usually derived from dietary fat and endogenous neonatal fats. When glycogen storage is saturated, ingesting glucose produces endogenous neonatal fat and promotes continuous triglyceride output and storage in adipose tissue [50-52]. In addition, obesity leads to excessive activation of lipolysis in adipose tissue, stimulating the release of fatty acids and leading to abnormal secretion of adipogenic factors, thus promoting the accumulation of ectopic fat and mild inflammation and aggravating insulin resistance [10].

Adipokines play important regulatory roles in insulin resistance, inflammatory response, and glucose and lipid metabolism [13]. Adiponectin is triggered to reduce fat by AMP protein kinase (AMPK) and through the higher peroxisome proliferator-activated receptor-alpha to increase the oxidation of fatty acids [53]. In addition, adiponectin also inhibits the expression of sterol reactive element binding protein $1 \mathrm{C}$ by inducing the LKB-AMPK pathway, thus downregulating the expression of downstream genes involved in fat generation and cholesterol synthesis [54]. As an appetiterelated adipose factor, leptin regulates food intake through the hypothalamus, which affects energy metabolism and body weight $[16,17]$. Leptin receptors are expressed in a variety of tissues, and their circulating level is proportional to the amount of fat. Studies have shown that leptin reduces the production of fat, increases the hydrolysis of triglycerides, and increases the oxidation of fatty acids [18]. Leptin also promotes the breakdown of fat in adipose tissue by increasing sympathetic nerve signaling [55]. Based on these studies, serum levels of adiponectin, leptin, and resistin were measured, and serum leptin and resistin levels increased significantly $(p<0.01)$, whereas adiponectin levels decreased significantly under the Western diet $(p<0.01)$. The level of adiponectin increased significantly $(p<0.01)$ after supplementation with desensitizers, and the levels of leptin and resistin decreased significantly $(p<0.01$; Figures 3(a)-3(c)).

Abnormal secretion of adipokines damages the storage of lipids in adipose tissue, leading to the heterotopic deposition of lipids, eventually inducing a series of chronic metabolic diseases $[8,9]$. In this study, we observed proliferation of histopathological areas of eWAT, white fat in the groin, and brown fat by H\&E staining and quantitatively analyzed the mean area of the three kinds of tissue cells. The results show that the cell area of the three types of adipocytes increased significantly under the Western diet $(p<0.01)$, and proliferation of the three types of adipocytes was significantly inhibited after administration of SDG $(p<0.01$; Figures 4(a)-4(c)).
Although the positive effects of SDG in improving obesity and regulating lipid metabolic disorder have been clearly reported, few reports are available on the molecular mechanisms [37-39]. In this experiment, gene expression related to lipid synthesis and lipid oxidative decomposition was analyzed, and SDG significantly inhibited the mRNA expression of lipogenic genes, such as Srebp1c, Fasn, Ppar, and Acaca $(p<0.01$; Figures $5(\mathrm{a})-5(\mathrm{~d}))$, and promoted mRNA expression of lipolytic genes, such as Ppar $\alpha$ and $\operatorname{Pgc1} \alpha(p<0.01$; Figures 5(e) and 5(f)). These results indicate that SDG regulates obesity and lipid metabolic disorder caused by a Western diet by regulating the expression of genes related to fat synthesis and decomposition. However, the further mechanism of action remains to be further studied.

In summary, SDG effectively inhibited weight gain, regulated the secretion of adipogenic factors, and suppressed the synthesis and accumulation of fat by regulating the expression of lipid synthesis and decomposition-related genes, which improved the obesity caused by a Western diet.

\section{Conclusion}

In this study, SDG effectively alleviated obesity and regulated lipid metabolic disorder. Improved obesity in response to SDG was preliminarily determined by suppression of body and tissue weight gains. Adipose tissue hyperplasia was further observed by $H \& E$ staining and adipocyte area was quantified, leading us to conclude that SDG inhibited hyperplasia of adipose tissue. In addition, SDG regulated the secretion of adipokines to achieve a balanced state. SDG inhibited the expression of lipogenic genes and promoted the expression of lipolytic genes.

\section{Data Availability}

Data were analyzed or generated during the study. The authors have conducted a lot of experiments where the data come from. The data used to support the findings of this study are available from the corresponding author upon request.

\section{Conflicts of Interest}

The authors declare no conflicts of interest.

\section{Authors' Contributions}

Shan Dong designed the study and developed methodology; Shan Dong, Wanli Sheng, and Ronghu Feng conducted the experiments; Wenliang Bai and Ronghu Feng did statistical analysis; Li Zhang edited the article.

\section{Acknowledgments}

This research was funded by Science, Technology and Innovation Commission of Shenzhen Municipality (grant number JCYJ20180306172311983). 


\section{References}

[1] S. Roossner, "Obesity: the disease of the twenty-first century," International Journal of Obesity, vol. 4, no. S4, pp. S2-S4, 2002.

[2] R. S. Cassani, P. G. Fassini, J. H. Silvah, C. M. Lima, and J. S. Marchini, "Impact of weight loss diet associated with flaxseed on inflammatory markers in men with cardiovascular risk factors: a clinical study," Journal of Nutrition, vol. 15, no. 1, p. 59, 2015.

[3] C. M. Steppan, S. T. Bailey, S. Bhat et al., "The hormone resistin links obesity to diabetes," Nature, vol. 409, no. 6818, pp. 307-312, 2001.

[4] Y. S. Mi, G. Y. Qi, Y. Gao et al., "EGCG ameliorates insulin resistance and mitochondrial dysfunction in hepg2 cells: involvement of bmal1," Molecular Nutrition \& Food Research, vol. 61, no. 12, Article ID 1700440, 2017.

[5] M. Blüher, "Obesity: global epidemiology and pathogenesis," Nature Reviews Endocrinology, vol. 15, no. 5, pp. 288-298, 2019.

[6] G. S. Hotamisligil, P. Arner, J. F. Caro, R. L. Atkinson, and B. M. Spiegelman, "Increased adipose tissue expression of tumor necrosis factor-alpha in human obesity and insulin resistance," Journal of Clinical Investigation, vol. 95, no. 5, pp. 2409-2415, 1995.

[7] D. M. Muoio and C. B. Newgard, "Obesity-related derangements in metabolic Regulation," Annual Review of Biochemistry, vol. 75, pp. 367-401, 2006.

[8] G. D. Pergola and F. Silvestris, "Obesity as a major risk factor for cancer," Journal of Obesity, vol. 2013, Article ID 291546, 11 pages, 2013.

[9] L. R. Howe, K. Subbaramaiah, C. A. Hudis, and A. J. Dannenberg, "Molecular pathways: adipose inflammation as a mediator of obesity-associated cancer," Clinical Cancer Research, vol. 19, no. 22, pp. 6074-6083, 2013.

[10] B. Kálmán and R. Michael, "Energy metabolism of white adipose tissue and insulin resistance in humans," European Journal of Clinical Investigation, vol. 48, no. 11, Article ID e13017, 2018.

[11] J. M. Olefsky and C. K. Glass, "Macrophages, inflammation, and insulin resistance," Annual Review of Physiology, vol. 72, no. 1, pp. 219-246, 2010.

[12] G. S. Heyn, L. H. Corrêa, and K. G. Magalhães, "The impact of adipose tissue-derived miRNAs in metabolic syndrome, obesity, and cancer," Frontiers in Endocrinology, vol. 11, Article ID 563816, 2020.

[13] N. Hosogai, A. Fukuhara, K. Oshima et al., "Adipose tissue hypoxia in obesity and its impact on adipocytokine dysregulation," Diabetes, vol. 56, no. 4, pp. 901-911, 2007.

[14] A. H. Berg, T. P. Combs, X. Du, M. Brownlee, and P. E. Scherer, "The adipocyte-secreted protein Acrp30 enhances hepatic insulin action," Nature Medicine, vol. 7, no. 8, pp. 947-953, 2001.

[15] P. E. Scherer, "Adipose tissue: from lipid storage compartment to endocrine organ," Diabetes, vol. 55, no. 6, pp. 1537-1545, 2006.

[16] J. M. Friedman and J. L. Halaas, "Leptin and the regulation of body weight in mammals," Nature, vol. 395, no. 6704, pp. 763-770, 1998.

[17] J. M. Montez, A. Soukas, E. Asilmaz, and G. F. Fayzikhodjaeva, "Acute leptin deficiency, leptin resistance, and the physiologic response to leptin withdrawal," Proceedings of the National Academy of Sciences of the United States of America, vol. 102, no. 7, pp. 2532-2542, 2005.
[18] W. N. William Jr., R. B. Ceddia, and R. Curi, "Leptin controls the fate of fatty acids in isolated rat white adipocytes," Journal of Endocrinology, vol. 175, no. 3, pp. 735-744, 2002.

[19] C. Rice-Evans, "Plant polyphenols: free radical scavengers or chain-breaking antioxidants?" Biochemical Society Symposia, vol. 61, pp. 103-116, 1995.

[20] J. Avila, J. R. García, G. Aguilar, and L. Rosa, “The antidiabetic mechanisms ofpolyphenols related to increased glucagon-like peptide-1 (GLP1) and insulinsignaling," Molecules, vol. 22, no. 6, p. 903, 2017.

[21] S. S. Deshpande, S. K. Sathe, and D. K. Salunkhe, "Chemistry and safety of plant polyphenols," Advances in Experimental Medicine and Biology, vol. 177, pp. 457-495, 1984.

[22] L. Vámos-Vigyázó, "Polyphenol oxidase and peroxidase in fruits and vegetables," Critical Reviews in Food Science and Nutrition, vol. 15, no. 1, pp. 49-127, 1981.

[23] E. Haslam, T. Lilley, Y. Cai, R. Martin, and D. Mangnolato, "Traditional herbal medicines-the role of polyphenols," Planta Medica, vol. 55, no. 1, pp. 1-8, 1989.

[24] R. J. Nijveldt, E. van Nood, D. E. van Hoorn, P. G. Boelens, K. van Norren, and P. A. van Leeuwen, "Flavonoids: a review of probable mechanisms of action and potential applications," The American Journal of Clinical Nutrition, vol. 74, no. 4, pp. 418-425, 2001.

[25] C. Liang, M. E. Oest, and M. R. Prater, "Intrauterine exposure to high saturated fat diet elevates risk of adult-onset chronic diseases in C57BL/6 mice," Birth Defects Research Part B: Developmental and Reproductive Toxicology, vol. 86, no. 5, pp. 377-384, 2009.

[26] L. K. Stewart, J. L. Soileau, D. Ribnicky et al., "Quercetin transiently increases energy expenditure but persistently decreases circulating markers of inflammation in C57BL/6J mice fed a high-fat diet," Metabolism, vol. 57, pp. S39-S46, 2008.

[27] L. Rivera, R. Morón, M. Sánchez, A. Zarzuelo, and M. Galisteo, "Quercetin ameliorates metabolic syndrome and improves the inflammatory status in obese zucker rats," Obesity, vol. 16, no. 9, pp. 2081-2087, 2008.

[28] A. S. Strimpakos and R. A. Sharma, "Curcumin: preventive and therapeutic properties in laboratory studies and clinical trials," Antioxidants \& Redox Signaling, vol. 10, no. 3, pp. 511-546, 2008.

[29] A. Asai and T. Miyazawa, "Dietary curcuminoids prevent high-fat diet-induced lipid accumulation in rat liver and epididymal adipose tissue," The Journal of Nutrition, vol. 131, no. 11, pp. 2932-2935, 2001.

[30] W. J. Spuyvander and E. Pretorius, "Is the use of resveratrol in the treatment and prevention of obesity premature?" Nutrition Research Reviews, vol. 22, no. 2, pp. 111-117, 2009.

[31] J. Burns, T. Yokota, H. Ashihara, M. E. J. Lean, and A. Crozier, "Plant foods and herbal sources of resveratrol," Journal of Agricultural and Food Chemistry, vol. 50, no. 11, pp. 33373340, 2002.

[32] U. Stervbo, O. Vang, and C. Bonnesen, "A review of the content of the putative chemopreventive phytoalexin resveratrol in red wine," Food Chemistry, vol. 101, no. 2, pp. 449-457, 2007.

[33] S.-J. Cho, U. J. Jung, and M.-S. Choi, "Differential effects of low-dose resveratrol on adiposity and hepatic steatosis in dietinduced obese mice," British Journal of Nutrition, vol. 108, no. 12, pp. 2166-2175, 2012.

[34] S. Kim, Y. Jin, Y. Choi, and T. Park, "Resveratrol exerts antiobesity effects via mechanisms involving down-regulation of 
adipogenic and inflammatory processes in mice," Biochemical Pharmacology, vol. 81, no. 11, pp. 1343-1351, 2011.

[35] M. Lagouge, C. Argmann, Z. Gerhart-Hines et al., "Resveratrol improves mitochondrial function and protects against metabolic disease by activating SIRT1 and PGC-1 $\alpha$," Cell, vol. 127, no. 6, pp. 1109-1122, 2006.

[36] S. Rom, V. Zuluaga-Ramirez, N. L. Reichenbach et al., "Secoisolariciresinol diglucoside is a blood-brain barrier protective and anti-inflammatory agent: implications for neuroinflammation," Journal of Neuroinflammation, vol. 27, no. 15 , p. $25,2018$.

[37] J. L. Adolphe, S. J. Whiting, B. H. J. Juurlink, L. U. Thorpe, and J. Alcorn, "Health effects with consumption of the flax lignan secoisolariciresinol diglucoside," British Journal of Nutrition, vol. 103, no. 7, pp. 929-938, 2010.

[38] S. Jian, Y. Tang, Y. Xiao et al., "Flaxseed lignans alleviate high fat diet-induced hepatic steatosis and insulin resistance in mice: potential involvement of AMP-activated protein kinase," Journal of Functional Foods, vol. 24, pp. 482-491, 2016.

[39] L. Sun, Y. Wang, Y. Song et al., "Resveratrol restores the circadian rhythmic disorder of lipid metabolism induced by high-fat diet in mice," Biochemical and Biophysical Research Communications, vol. 458, no. 1, pp. 86-91, 2015.

[40] Y. Wang, B. Fofana, M. Roy et al., "Flaxseed lignan secoisolariciresinol diglucoside improves insulin sensitivity through upregulation of GLUT4 expression in diet-induced obese mice," Journal of Functional Foods, vol. 18, pp. 1-9, 2015.

[41] C. C. Yu, "Effects of oil preparation technology on the content of phenolic compounds and their antioxidant properties in flaxseed cake," Master's thesis, Jiangnan University, Wuxi, China, 2019.

[42] Y. Zhao, "Functional components of flaxseed and its application value in food industry," ShanXi Food Industry, vol. 2, pp. 31-33, 2005.

[43] C. Dzuvor, J. Taylor, C. Acquah, S. Pan, and D. Agyei, "Bioprocessing of functional ingredients from flaxseed," Molecules, vol. 23, no. 10, p. 2444, 2018.

[44] A. N. Martinchik, A. K. Baturi, V. V. Zubtsov, and V. I. Molofeev, "Nutritional value and functional properties of flaxseed," Voprosy Pitaniia, vol. 81, no. 3, pp. 4-10, 2012.

[45] S. Fukumitsu, K. Aida, N. Ueno, S. Ozawa, Y. Takahashi, and M. Kobori, "Flaxseed lignan attenuates high-fat diet-induced fat accumulation and induces adiponectin expression in mice," British Journal of Nutrition, vol. 100, no. 3, pp. 669-676, 2008.

[46] W. Zhang, X. Wang, Y. Liu et al., "Dietary flaxseed lignan extract lowers plasma cholesterol and glucose concentrations in hypercholesterolaemic subjects," British Journal of Nutrition, vol. 99, no. 6, pp. 1301-1309, 2008.

[47] J. Kang, J. Park, H.-L. Kim et al., "Secoisolariciresinol diglucoside inhibits adipogenesis through the AMPK pathway," European Journal of Pharmacology, vol. 820, pp. 235244, 2018.

[48] A. Pan, W. Demark-Wahnefried, X. Ye et al., "Effects of a flaxseed-derived lignan supplement on C-reactive protein, IL6 and retinol-binding protein 4 in type 2 diabetic patients," British Journal of Nutrition, vol. 101, no. 8, pp. 1145-1149, 2009.

[49] E. W. Gregg, Y. J. Cheng, B. L. Cadwell et al., "Secular trends in cardiovascular disease risk factors according to body mass index in US adults," Journal of American Medical Associations, vol. 293, no. 15, pp. 1868-1874, 2005.
[50] S. M. Grundy, "Adipose tissue and metabolic syndrome: too much, too little or neither," European Journal of Clinical Investigation, vol. 45, no. 11, pp. 1209-1217, 2015.

[51] F. B. Hillgartner, L. M. Salati, and A. G. Goodridge, "Physiological and molecular mechanisms involved in nutritional regulation of fatty acid synthesis," Physiological Reviews, vol. 75, no. 1, pp. 47-76, 1995.

[52] R. Gao, Q. Yu, Y. Shen et al., "Production, bioactive properties, and potential applications of fish protein hydrolysates: developments and challenges," Trends in Food Science \& Technology, vol. 110, pp. 687-699, 2021.

[53] T. Yamauchi, J. Kamon, Y. Ito et al., "Cloning of adiponectin receptors that mediate antidiabetic metabolic effects," Nature, vol. 423, no. 6941, pp. 762-769, 2007.

[54] M. Awazawa, K. Ueki, K. Inabe et al., "Adiponectin suppresses hepatic SREBP1c expression in an AdipoR1/LKB1/AMPK dependent pathway," Biochemical \& Biophysical Research Communications, vol. 382, no. 1, pp. 51-56, 2009.

[55] T. J. Bartness, C. K. Song, H. Shi, R. R. Bowers, and M. T. Foster, "Brain-adipose tissue cross talk," Proceedings of the Nutrition Society, vol. 64, no. 1, pp. 53-64, 2005. 